\title{
Estructura y la ultraestructura del ovario de Cichlasoma urophthalmus (Perciformes: Cichlidae)
}

\author{
Rubí Viedma ${ }^{1}$, Jonathan Franco ${ }^{2}$, Carlos Bedia ${ }^{1}$, Guadalupe Guedea Fernández ${ }^{1}$, Héctor Barrera \\ Villa Zevallos ${ }^{3} \&$ Héctor Barrera Escorcia ${ }^{1 *}$ \\ 1. Laboratorio de Microscopia, Facultad de Estudios Superiores Iztacala, U.N.A.M. C.P. 54090, Estado de México; \\ araceliviedma@hotmail.com, bediacharly@yahoo.com.mx, dalethguedea@hotmail.com, hectorbarrerae@hotmail.com \\ 2. Laboratorio de Ecologia, Facultad de Estudios Superiores Iztacala, U.N.A.M. C.P. 54090, Estado de México; \\ jonfrancol@yahoo.com \\ 3. Consejo Nacional de Ciencia y Tecnología, Av. Insurgentes Sur 1582, Col. Crédito Constructor Del. Benito Juárez C.P. \\ 03940, México, D.F.; hectorbarrerav@hotmail.com \\ * Correspondencia
}

Recibido 04-VI-2010. C Corregido 03-X-2010. Aceptado 04-XI-2010.

\begin{abstract}
Structure and ultrastructure of the ovary of Cichlasoma urophthalmus (Osteichthyes: Cichlidae). The study of the normal development, differentiation, structure and function of various components of developing follicles in the ovaries of numerous fish species have been a consistent focus of comparative reproduction. The structural and ultrastructural features of gonads from Cichlasoma urophthalmus have received scarce attention. In this work, we realized a descriptive study of female gonads of Cichlasoma urophthalmus. A total of 40 samples were collected in the Veracruz Alvarado Lagoon, Mexico in 2007-2008 period including the windy, dry and rainy seasons. Female gonads were extracted and a portion was fixed in $4 \%$ formaldehyde for treatment for routine histology hematoxylin and eosin (HE) and another part was processed for transmission electron microscopy (TEM). The gonads were fixed in $3 \%$ glutaraldehyde and $2 \%$ osmium tetroxide, followed by dehydrated in ethanol 50\%, 70\%, 80\%, 95\% and 100\% for inclusion in Epon, thin sections were then prepared and were contrasted with lead citrate and uranyl acetate. The process of oocyte development can be divided into five distinct stages (formation of oocytes from oogonia, primary growth, lipid stage, vitellogenesis and maturation). In this work, we found that the primary growth stage is characterized by intense RNA synthesis and the differentiation of the vitelline envelope. Secondary growth starts with the accumulation of lipid droplets in the oocyte cytoplasm (lipid stage), which is then followed by massive uptake and processing of proteins into yolk platelets (vitellogenic stage). During the maturation stage, the lipid inclusions coalesce into a single oil droplet, and hydrolysis of the yolk platelets leads to the formation of a homogeneous mass of fluid yolk in mature eggs. In conclusion, further studies should elucidate structure and ultrastructural changes in the ovarian follicular components, in C. urophthalmus during different stages of oocyte growth. Rev. Biol. Trop. 59 (2): 743-750. Epub 2011 June 01.
\end{abstract}

Key words: C. urophthalmus, ovaries, estructure and ultraestructure, oocyte.

El pez Cichlasoma urophthalmus (Günter 1862) es una especie comestible de consumo local comúnmente conocida como "Mojarra prieta" o "Mojarra del Sureste", que se puede capturar durante todo el año, con reproducción entre los meses de Marzo a Octubre. Esta especie es endémica de la República Mexicana, se localiza en el Río Usumacinta, Península de Yucatán, Isla Mujeres y en la Laguna de Alvarado en el Estado de Veracruz. En cuanto a la biología reproductiva de $C$. urophthalmus presenta un dimorfismo sexual visible entre machos y hembras: los machos son más delgados y largos, y presentan unas aletas más desarrolladas 
y menos redondeadas que una hembra adulta (Bedia \& Franco 2008). Sin embargo, no se desconoce el detalle de la biología reproductiva de $C$. urophthalmus, por lo que resulta de gran interés el estudio de la diferenciación, la estructura y la función del desarrollo folicular de los ovarios. Esto permitirá no sólo conocer el estado reproductivo de las hembras, sino que hará posible diseñar y mejorar propuestas de gestión para las especies de importancia económica en la zona (Guraya 1986, Cruz-Landim \& CruzHöfling 2001, Muñoz et al. 2002, Vitale et al. 2005). Por lo tanto, el presente trabajo tiene la finalidad de comparar la morfología y la ultraestructura de las células germinales de $C$. urophthalmus, basado en estudios histológicos y de microscopia electrónica de transmisión.

\section{MATERIAL Y MÉTODOS}

Se recolectaron 40 ejemplares de $C$. urophthalmus en la Laguna de Alvarado (Veracruz), localizada a los $18^{\circ} 59^{\prime} \mathrm{N}$ y los $19^{\circ} 00^{\prime} \mathrm{N}$ - 9557' W y 9600' W, en el período 2007-2008 que incluyó las temporadas de Nortes, Secas y Lluvias. Los ejemplares fueron recolectados por medio de una red de $40 \mathrm{~m}$ de largo y $2.5 \mathrm{~m}$ de alto y malla de $2.5 \mathrm{~cm}$ de acuerdo con ArceoCarranza et al. 2004. Los organismos fueron anestesiados con Tricaína metano sulfonato (MS222), posteriormente, los ovarios fueron removidos y fijados en formol al $4 \%$. Las muestras fueron preparadas para microscopía óptica, las cuales fueron lavadas y deshidratadas en alcoholes graduales para su inclusión en parafina con índice de inclusión de $56^{\circ} \mathrm{C}-58^{\circ} \mathrm{C}$. Luego, se hicieron cortes en secciones longitudinales, en un micrótomo de rotación (American Optical Mod 820) a $7 \mu \mathrm{m}$ de espesor, las preparaciones fueron teñidas por la técnica histológica de rutina y montadas en resina sintética (Luna 1958). Las preparaciones histológicas obtenidas fueron analizadas en un microscopio óptico Nikon Eclipse mod. E-400 y las fotos fueron tomadas en una cámara Sony-Ex wave HAD. Para microscopía electrónica los ovarios fueron fijados en glutaraldehído al 3\% amortiguado con buffer de fosfatos a $\mathrm{pH} 7.4$ frío por
$24 \mathrm{hr}$ a $4^{\circ} \mathrm{C}$, se lavaron con la misma solución amortiguadora y se pos-fijaron con tetróxido de osmio $\left(\mathrm{OsO}_{4}\right)$ al $2 \%$, y fueron lavados al final con la misma solución amortiguadora de fosfatos. Se realizó el proceso de deshidratación con etanol al 50\%, 70\%, 80\%, 95\% y finalmente dos cambios de etanol al $100 \%$. La infiltración fue realizada con una mezcla de óxido de propileno-epón 1:1 y Epón-Araldita; la polimerización fue realizada a $60^{\circ} \mathrm{C}$ durante $48 \mathrm{hr}$. Se obtuvieron cortes longitudinales en el ultramicrótomo de $800^{\circ} \mathrm{A}-1200^{\circ} \mathrm{A}$ para obtener cortes semifinos los cuales, fueron teñidos con azul de Toluidina y finos a $600 \mathrm{~A}^{\circ}$ que fueron contrastados con acetato de uranilo y citrato de plomo (Sjöstrand 1971, Cárdenas \& Barrera 1998) posteriormente las muestras fueron observadas en un microscopio electrónico de transmisión Jeol mod. 1200 EX 2.

\section{RESULTADOS}

Ovarios: Los ovarios de C. urophthalmus son pareados en forma de sacos huecos, ubicados en la cavidad pleuroperitoneal, dorsales en relación al intestino y ventrales al riñón. Fusionados caudalmente, forman un corto oviducto que desemboca en el poro genital (Bedia $\&$ Franco 2008) (Fig. 1A). Los ovarios de $C$. urophthalmus contienen ovocitos de diferentes estados de desarrollo que incluyen: Estado Perinuclear, Estado Alvéolo Cortical, Estado Previtelogénico y Estado Vitelogénico (West 1990). El ovario contiene dos tipos de células, somáticas y germinales. Las ovogonias están dispuestas en forma discontinua y pueden realizar divisiones mitóticas o iniciar la división meiótica para formar varios ovocitos, por lo tanto el desarrollo de los folículos ocurre en dos etapas, previtelogénicos y vitelogénicos (Fig. 1B). (Grier 2000). En el ovario se extienden numerosos pliegues ovígeros revestidos por epitelio germinal de células planas o cúbicas (Fig. 2 A y B).

Ovogonias: Son células ovoides que miden aproximadamente de 8 a $10 \mu \mathrm{m}$, presentan un núcleo prominente de 5 a $6 \mu \mathrm{m}$ de 

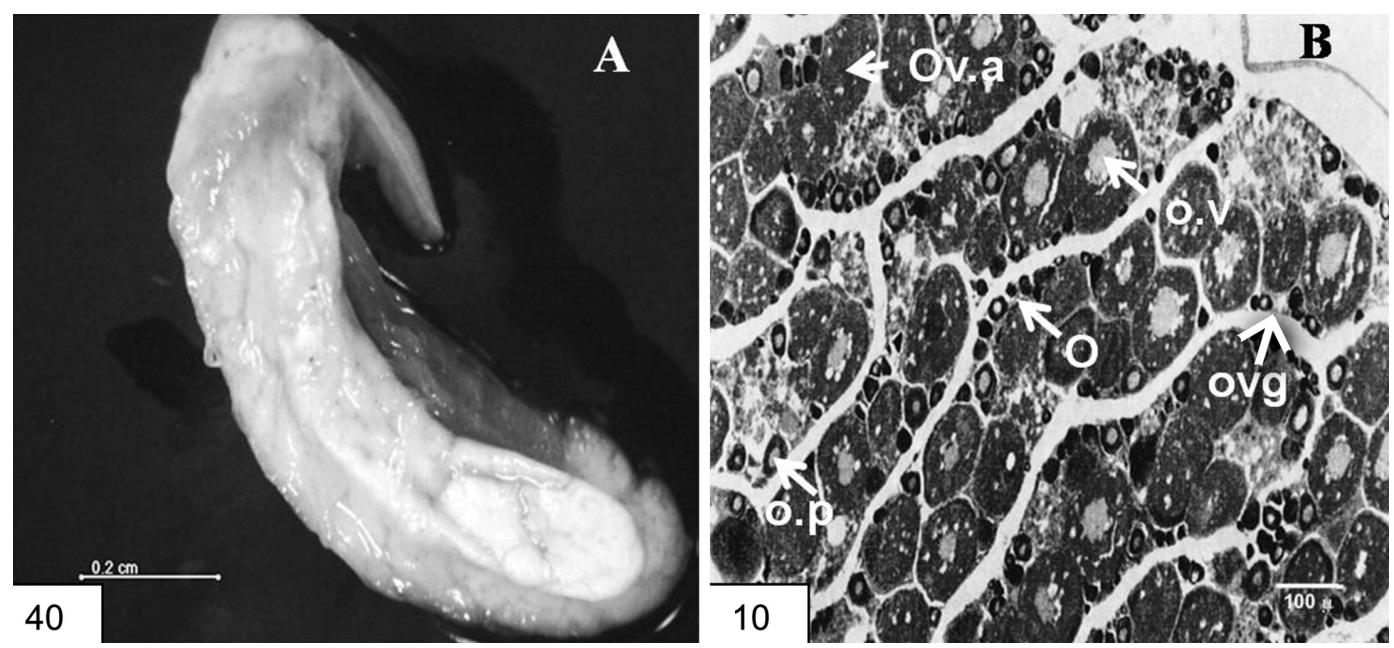

Fig. 1. (A). Morfología del ovario de Cichlasoma uropthalmus (B). Corte transversal de 7 micrómetros teñido con $\mathrm{H}$ y E del ovario donde se observa un desarrollo asincrónico: ovocito atrésico Ov.a, ovocito vitelogénico Ov, ovocito previtelogénico o.p, pliegue ovígero ovg, ovogonia $\mathbf{O}$.

Fig. 1. (A). Ovarian Morphology of Cichlasoma uropthalmus (B). Cross section of 7 micrometers with H \& E stained ovary where there has an asynchronous development: atretic oocytes Ov.a, previtellogenic oocyte o.p, vitellogenic oocyte $\mathbf{O v}$, ovigerous fold ovg, oogonia $\mathbf{O}$.

diámetro y su posición es central, con 1 a 4 nucléolos en su interior, escaso citoplasma, teñido débilmente. Las ovogonias se observan rodeadas de epitelio germinativo ovárico.

Ovocitos perinucleolares: Miden aproximadamente 40 a $80 \mu \mathrm{m}$, presentan un citoplasma fuertemente basófilo, al final de este estadio se pierde gradualmente su naturaleza basófila, son más redondos y los nucléolos empiezan a migrar hacia la periferia (Fig. 2 C).

Ovocitos alveólo-corticales: Estos ovocitos miden aproximadamente $100 \mu \mathrm{m}$ al comienzo de esta etapa, se observa una capa simple de células aplanadas que envuelve al ovocito, durante el desarrollo se transforman a cúbicas y se inicia la formación de la zona pelúcida, entre el ovocito y las células foliculares. En esta etapa, los alvéolos corticales inicialmente son pocos y tienen distribución homogénea en el citoplasma. Posteriormente, incrementan su número y se ubican alrededor del núcleo (perinucleares), y se empiezan a formar los gránulos de vitelo (Fig. 2 D).

Ovocitos previtelogénicos: Durante esta etapa se observaron ovocitos previtelogénicos de tamaño pequeño de 110 a $180 \mu \mathrm{m}$, asociados a los pliegues ovígeros, que presentan un núcleo grande en relación al volumen citoplásmico, muestran una fuerte afinidad por la hematoxilina, debido a la presencia de un aumento de organelos celulares como retículo endoplásmico, ribosomas y mitocondrias, que le dan la característica al ovoplasma de ser basófilo (Uribe et al. 2006) (Fig. 2 F). Durante el desarrollo de los ovocitos se observó un aumento en su tamaño, debido a la acumulación de grasas y proteínas, por lo que se presenta un cierto grado de zonación con respecto a la disposición del vitelo, y también se pudo observar la aparición de nucléolos en la periferia de núcleo y con ello un incremento en la cantidad de ribosomas en el ooplasma; en esta etapa no se distinguió la zona radiada. 

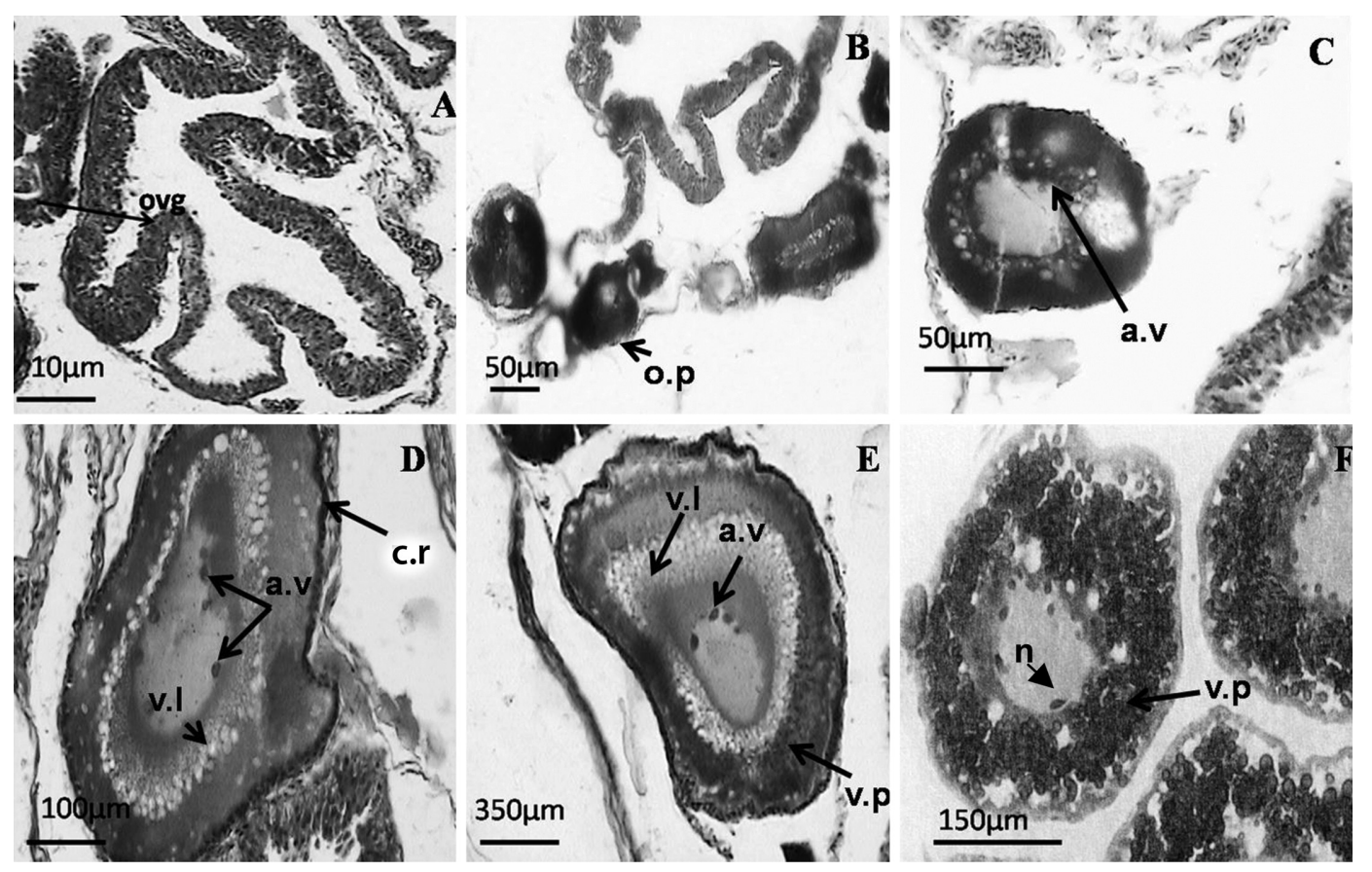

Fig. 2. Cortes histológicos de 7 micrometros (A) pliegues ovígeros ovg con algunas células epiteliales, (B) ovocitos previtelogénicos o.p con afinidad por hematoxilina (C) ovocito en etapa de alveolo cortical adherido a la lamela ovígera (D) ovocito perinucleolar con inicios de vitelogénesis todavía unidos a la lamelas ovígeras en donde se observa corticales alveolos a.v, vacuolas lípidicas v.I y corion c.r (E) ovocito vitelogénico con acumulación de capas concéntricas de proteínas v.p y lípidos v.I que rodean al núcleo (F) ovocito vitelogénico maduro con algunos nucleólos periféricos $\mathbf{n}$.

Fig. 2. Histological sections of 7 micrometers (A) ovigerous folds with some epithelial cells, (B) oocytes with an affinity for hematoxylin (C) oocytes in cortical alveoli stage attached to the ovigerous lamella (D) with early perinucleolar oocyte vitellogenesis still attached to the ovigerous lamellae where is observed cortical alveoli a.v, lipid vacuoles v.I and chorion c.r (E) vitellogenic oocyte with accumulation of concentric layers of proteins v.p and lipids v.I that surround the nucleus (F) mature oocytes with some peripheral nucleoli $\mathbf{n}$.

Ovocitos vitelogénicos: Durante la vitelogénesis el ovocito de $C$. urophthalmus tiene un tamaño de $350-600 \mu \mathrm{m}$, y en esta etapa se acumulan sustancias de reserva que constituyen al vitelo, como es la acumulación de capas de proteínas y lípidos que se disponen centrípetamente al núcleo, dándole una apariencia esponjosa (Fig. 2 E). Durante la vitelogénesis, las células foliculares tienen una actividad muy importante, en la captación y 1 selección de múltiples sustancias del exterior provenientes de los vasos sanguíneos. En esta etapa se hace más evidente la zona radiada de $C$. urophthalmus, la cual está compuesta por dos capas; la más delgada y densa es la que está en contacto con las células foliculares (Fig. 3 A y F), y la otra capa, que está en contacto con la membrana plasmática, es más gruesa y con estriaciones más visibles, por lo que corresponde a una estructura bipartita.

Ovocitos atrésicos: Los ovocitos de este estadio disminuyen de tamaño significativamente y muestran una forma irregular. La zona pelúcida presentó un mayor grado de fragmentación y disolución. La teca vascularizada se observó subyacente entre plegamientos de las células foliculares (Fig. $1 \mathrm{~A}$ ). 

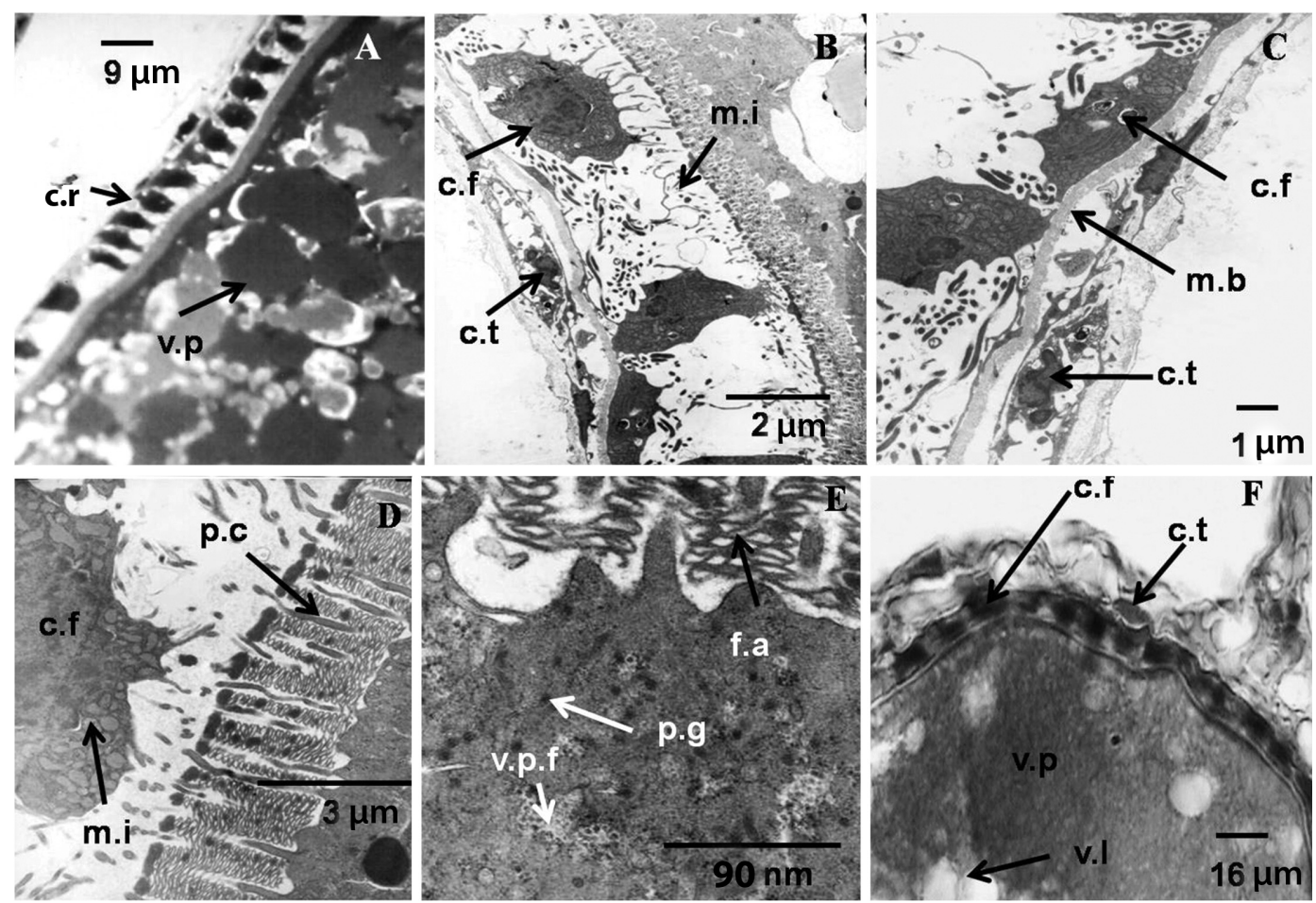

Fig. 3 A y F. Cortes semifinos incluidos en epon de 7 micrometros teñidos con azúl tripano al microscopio óptico en donde se observan: el corion c.r y algunas vesículas proteicas v.p, las células foliculares c.f, las células de la teca c.t y algunas vesículas proteicas v.p y lipídicas v.l. (B) corte al microscopio electrónico de las células foliculares pasando material de intercambio m.i por medio de las microvellosidades al ovocito y células de la teca c.t en la parte periférica (C) se observan 2 células foliculares c.f cercanas a la membrana basal $\mathbf{m}$.b y una célula de la teca c.t (D) se observa una célula folicular c.f con abundante material de intercambio m.i a través de sus prolongaciones citoplásmicas p.c (E) se observan algunos gránulos pigmentados obscuros p.g en el citoplasma del ovocito junto con algunas vesículas proteicas en formación v.p.f, en la parte superior de la fotografía algunas fibras de actina f.a que conforman los canales de poro.

Fig. $3 \mathrm{~A}$ and F. Semi cuts included in epon 7 micrometers stained with trypan blue under light microscopy where note: The radiate area z.r, and some protein vesicles v.p, follicular cells c.f, theca cells c.t and some protein and lipid vesicles v.p and $\mathbf{v . l}$, (B) shows a cut in the electron microscope of the follicle cells exchange material $\mathbf{m} . \mathbf{i}$ through the microvilli to the oocyte and theca cells c.t in the peripheral part, (C) shows two follicular cells c. $\mathbf{f}$ near the basement membrane $\mathbf{m} . \mathbf{b}$ and a theca cell c.t (D) shows a follicular cell c.f with abundant material exchange m.i through cytoplasmic extensions p.c in Figure $\mathrm{E}$ are seen some dark pigmented granules p.g in the cytoplasm of the oocyte near to some protein vesicles v.p.f. in the top of the picture some actin fibers f.a that form the channel pore.

Cubiertas ovocitarias: Las células de la teca se ubican por fuera del epitelio folicular y rodean completamente al ovocito. Estas células están rodeadas por tejido conectivo, fibroblastos y vasos sanguíneos, y en este estadio, las células tecales presentan mitocondrias pleomórficas (Nichols \& Mapple 1972, Gresik et al.1973) (Fig. 3 B y C). Están dispuestas como una monocapa que se encuentra en contacto directo con las células foliculares, las cuales se interdigitan con el ovocito para incrementar el área superficial de absorción y así favorecer el intercambio de metabolitos por pinocitosis. También se observan algunas proyecciones dirigidas hacia la zona del corion, y algunas fibras de actina, que conforman los canales de poro, lugar por donde gran cantidad de material orgánico es transferido 
al ovocito (Fig. 3 D y E). Algunos materiales de intercambio en el citoplasma del ovocito son desde muy laxos hasta algunos de carácter electrodenso, probablemente se trata de glicoproteínas y una gran cantidad de pequeños cuerpos pigmentados que presumiblemente viajan desde la zona de la teca y la granulosa, hasta el interior del ovocito (Fig. 3 E).

\section{DISCUSIÓN}

El desarrollo folicular del ovario es el resultado de procesos complejos de desarrollo y diferenciación controlados por hormonas, los cuales involucran cambios celulares del ovocito (Guraya 1986). En este trabajo el crecimiento inicial de los ovocitos se caracteriza por la presencia de un núcleo que sufre constantes transformaciones como el incremento del número de nucléolos que tienen como función la biosíntesis de ribosomas y con ello la formación de ARN ribosomal (Goetz \& Garcynski 1997), los cuáles son necesarios para la biosíntesis de proteínas celulares en los ovocitos. La presencia de los gránulos de vitelo y material electrodenso se debe a la abundante cantidad de proteínas y síntesis de ARN (Wallace \& Selman 1990, Muñoz et al. 2002). La acumulación de las gotas de lípidos es un indicador del inicio de la segunda fase de crecimiento del ovocito. En este trabajo se pudo determinar los componentes estructurales, durante estas fases de desarrollo, los ovocitos se desprenden de los pliegues ovígeros aún en distintos grados de maduración. Los ovocitos en estado previtelogénico con nucléolos periféricos muestran a diferencia de otras especies (Neidig et al. 2000) las gotas lipídicas, que inicialmente se colocan en la zona cercana al núcleo mientras que las vesículas proteicas aparecen poco después en la capa concéntrica superior y migran hacia la periferia de la célula, en este estado no se encontraron gotas de grasa fusionadas en la zona cercana al núcleo como en el caso de Centropomus undecimalis (Grier 2000). En el estado vitelogénico se observó una interacción e intercambio de materiales orgánicos entre las células de la teca y de la granulosa con el ovocito, debido a la presencia de numerosas microvellosidades, que comunican a las células foliculares con la membrana plasmática del ovocito, también se hizo evidente. Los ovarios de $C$. urophthalmus corresponden al tipo cistovárico y en su interior se distribuyen las láminas ováricas que contienen los folículos ováricos en distintos estadios de maduración. El proceso de crecimiento y maduración de los folículos ováricos de esta especie comprende tres procesos generales: (1) cambios nucleares con formación de numerosos nucleolos, (2) cambios citoplasmáticos con acumulación de vesículas vitelinas, gránulos vitelinos y vesículas lipídicas, y (3) desarrollo de la pared folicular (Selman \& Wallace 1983). Las células de la teca están compuestas de Tejido Conectivo y vasos sanguíneos con algunos desmosomas que interconectan las células. Trabajos previos proponen que estas células podrían producir precursores de esteroides (andrógenos) y las células granulosas podrían convertir andrógenos a $17 \beta$ estradiol u otros mediadores esteroideos (Nakamura \& Nagahama 1993, Nagahama 1997, Devlin \& Nagahama 2002, Cárdenas et al. 2008). Las células granulosas poseen organelos típicos de células secretoras de proteínas lo cual sugiere funciones adicionales en la síntesis de la cubierta y la producción de enzimas involucradas en la conversión de precursores de esteroides. En este trabajo se encontraron numerosas células tecales, lo cual puede indicar que esta especie presenta una gran capacidad de producción de esteroides. La mayoría de los Cíclidos durante su desarrollo gonadal, una maduración asincrónica, a diferencia de Nematogenys inermis que pertenece a la categoría de sincronismo parcial que tiene otras estrategias de desove (Huaquin et al. 2002). Es necesario seguir el proceso reproductivo de $C$. uruphthalmus para determinar además las etapas de desarrollo de la ovogénesis en las temporadas de desove (Prisco et al. 2002, Prisco et al. 2004). 


\section{AGRADECIMIENTOS}

Para Adolfo García Sáenz del Instituto de Fisiología Celular por el uso del microscopio electrónico de transmisión. Al programa PAPCA-2007-2008 de la FES-I, por apoyar el proyecto "La Laguna de Alvarado; Biodiversidad y dinámica trófica en dos hábitats críticos”.

\section{RESUMEN}

Se realizó un estudio descriptivo de las gónadas femeninas de Cichlasoma urophthalmus. Las muestras fueron recolectadas en la Laguna de Alvarado Veracruz, México en el período 2007-2008 que incluyó las temporadas de Nortes, Secas y Lluvias. Se extrajeron las gónadas femeninas y una parte se fijó en formol al $4 \%$ para su tratamiento por técnica histológica de rutina hematoxilina y Eosina (H-E) y otra parte se procesó para microscopia electrónica de transmisión. Las gónadas se fijaron en glutaraldehído al $3 \%$ y $\mathrm{OsO}_{4}$ al $2 \%$, se deshidrataron en etanol de 50 al $100 \%$ para ser incluidas en Epón. Se realizaron cortes finos y semifinos contrastados con citrato de plomo y acetato de uranilo. Los ovarios de C. urophthalmus son pareados presentan un desarrollo asincrónico con ovocitos previtelogénicos en estadio perinuclear tardío, asociados a las lamelas ovígeras y ovocitos vitelogénicos del VII estadio, éstos últimos presentan una zona radiada bien definida, con gránulos de vítelo lipídico y vesículas de vítelo proteico que se distribuyen en capas concéntricas, que rodean al núcleo. El presente estudio, permitió conocer más a fondo los cambios de la estructura y ultraestructura de los componentes de los folículos ováricos, en C. urophthalmus durante las diferentes etapas de crecimiento de los oocitos.

Palabras clave: $C$. urophthalmus, ovarios, estructura y ultraestructura.

\section{REFERENCIAS}

Arceo, D.J., J. Franco, G.L. Waggy \& R. Chávez. 2004. Trophic comparison of two species of needlefish (Belonidae) in the Alvarado Lagoonal System, Veracruz, Mexico. Gulf. Caribb. Res. 16: 81-88.

Bedia, C. \& J. Franco. 2008. Peces de los sistemas costeros del Estado de Veracruz. FES-Iztacala U.N.A.M. México, D.F.

Cárdenas, R. \& H. Barrera. 1998. Histología y ultraestructura del testículo del charal Chirostoma jordani (Osteichtyes: Ahterinidae). Rev. Biol. Trop. 46: 943-949.
Cárdenas, R., M. Chávez, J.L. González, P. Aley, J. Espinoza \& L.F. Jiménez. 2008. Oocyte structure and ultrastructure in the Mexican silverside fish Chirostoma humboldtianum (Atheriniformes: Atherinopsidae). Rev. Biol. Trop. 56: 1371-1380.

Cruz-Landim, C. \& M.A. Cruz-Höfling. 2001. Ultrastructure of ovarian follicular epithelium of the Amazonian fish Pseudotylosurus microps (Teleostei: Belonidae): morphological and histochemical characterization of intercellular deposits. Rev. Bras. Biol. 61: 133-140.

Devlin, R.H. \& Y. Nagahama.2002. Sex determination and sex differentiation in Fish: an overview of genetic, physiological, and environmental influences. Aquaculture 208: 191-364.

Goetz, F.W. \& M. Garcynski. 1997. The ovarian regulation of ovulation in teleost. Fish. Physiol. Biochem. 17: 33-38.

Gresik, E.W., G. Quirk \& J.B. Hamilton. 1973. Fine structure of the Sertoli cell of the testis of the teleost Oryzias latipes. Gen. Comp. Endocrinol. 21: 341-352.

Grier, H. 2000. Ovarian germinal epithelium and folliculogenesis in the common snook, Centropomus undecimalis (Teleostei: Centropomidae). J. Morphol. 243: 265-281.

Guraya, S.S. 1986. The cell and molecular biology of fish oogénesis. Monogr. Dev. Biol. 18: 117-125.

Günter, A.A. 1862. Catalogue of fishes in the British Museum. Catalogue of the Acanthopterygii, Paryngognathi and Anacanthini in the collection of the British Museum. American Museum of Natural History Library, Londres, Inglaterra.

Huaquín, L.G., D. Veliz \& G. Arratia. 2002. Estudio comparativo de ovarios y cubiertas ovocitarias en peces siluriformes de aguas continentales de Chile. Gayana Zool. 66: 269-274.

Luna, L.G. 1958. Manual of the Armed Forces institute of pathology. McGraw-Hill, Nueva York, EEUU.

Muñoz, M., M. Sabat, S. Mallol \& M. Casadevall. 2002. Gonadal structure and gametogenesis of Trigla lyra (Pisces:Triglidae). Zool. Stud. 41: 412-420.

Nagahama, Y. 1997. 17 $\alpha, 20 \beta$-dihydroxy-4-pregnen-3-one, a maturation-inducing hormone in fish oocytes: Mechanism of synthesis and action. Steroid. 62: 190-196.

Nakamura, M. \& Y. Nagahama. 1993. Ultrastructural study on the differentiation and development of steroid producing cells during ovarian differentiation in amago salmon, Oncorhynchus rhodurus. Rev. Aquaculture 112: $237-251$. 
Neidig, L.C., D.P. Skapura, H.J. Grier \& C.W. Dennis. 2000. Techniques for spawning common snook: broodstock. handling, oocyte stating and egg quality. N. Am. J. Aquacult. 62: 103-113.

Nichols, T.J \& G. Mapple. 1972. Ultrastructure observations on possible sites of steroids biosynthesis on the ovarian follicular epithelium of two species of cichlid fish, Cichlasoma nigrofasciatum and Haplochromis multicolor. Zellforch Mikrosk. Anat. 128: 317-335.

Prisco, M., L. Ricchiari \& P. Andreuccetti. 2002. Ultrastructural studies on developing follicles spotted ray Torpedo marmorata. Mol. Reprod. Dev. 61: 78-86.

Prisco, M., S. Valiant, M. Romano, L. Ricchiari, A. Liguoro, V. Laforgia, E. Limatola \& P. Andreucchetti. 2004. Ovarian follicle cells in Torpedo marmorata synthesis vitellogenin. Mol. Reprod. Dev. 67: 424-429.

Selman, K. \& R.A. Wallace. 1983. Oogenesis in Fundulus heteroclitus. III. Vitellogenesis. J. Exp. Zool. 226: 441-457.
Sjöstrand, F.S. 1971. Electron microscopy of cells and tissues. Academic, Nueva York, EEUU.

Uribe, A.C., A. García, S. Milena \& M. Aguilar. 2006. Características histológicas de los estudios de atresia de folículos ovaricos en dos especies de Teleósteos Vivíparos: Llyodon Whitei (Meek, 2002) y Godea atripinnis (Jordan 1998) (Goodeidae). Rev. Hydrobiol. Trop. 16: 67-73.

Vitale, F., M. Cardinale \& H. Svedeng. 2005. Evaluation of the temporal development of the ovaries in cod (Gadus morhua) from the Sound and Kattegat. J. Fish Biol. 67: 669-683.

Wallace, R.A. \& K. Selman. 1990. Ultrastructural aspects of oogenesis and oocyte growth in fish and amphibians. J. Electron. Microsc. Tech. 16: 175-201.

West, G. 1990. Methods of assessing ovarian development in Fishes: a review. Aust. J. Mar. Freshwat. Res. 41: 199-222. 